\title{
Comparison of endoscopic Vs microscopic ossiculoplasty; a study of 157 consecutive cases
}

\author{
Holli Coleman ${ }^{1}$, Theofano Tikka ${ }^{2}$, John Curran ${ }^{2}$, and Arunachalam Iyer ${ }^{2}$ \\ ${ }^{1}$ NHS Lanarkshire \\ ${ }^{2}$ Monklands Hospital
}

January 24, 2022

\begin{abstract}
1) Results of ossiculoplasty via trans-canal endoscopic (TEES) route is comparable to that of the traditional microscopic approach 2) There was no difference in complications or secondary outcomes such as tympanic membrane residual perforation or ear discharge between these groups 3) Both groups achieved an air-bone gap of less that 20dB in more than $70 \%$ of the cases 4) In our experience there was no difference in outcomes based on ossiculoplasty material such as cartilage, titanium prosthesis or autologous grafts such as incus. 5) Eroded stapes superstructure is does not preclude getting improvements in ABG of less than $20 \mathrm{~dB}$, but larger studies with longer follow ups might be useful.
\end{abstract}

Key Words: endoscopic, hearing loss, ossiculoplasty, tympanomastoidectomy, tympanoplasty

Level of evidence: 3

\section{Introduction}

Trans-canal endoscopic ear surgery (TEES) carries the benefits of enhanced visualization shorter operating times and no external incision. However, TEES does have the disadvantage of being a one-handed procedure and bleeding can jeopardize the visual field ${ }^{\mathbf{1}}$. Current literature suggests TEES provides similar audiometric, functional, and safety outcomes to those of microsurgery in tympanoplasty, cholesteatoma surgery and stapedectomy ${ }^{\mathbf{2 , 3}}$. There are very few published studies on outcomes of TEES in ossiculoplasty. Our study aimed to compare audiometric outcomes of patients undergoing ossiculoplasty employing either the TEES or microsurgery approach performed by a single otologist.

\section{Methods}

\subsection{Study Design}

Retrospective analysis of data from a prospective audit of ossiculoplasty. STROBE guidelines were used to present the findings.

\subsection{Setting}

Patients underwent surgery in a secondary care hospital between 2009-2018

\subsection{Participants}

157 consecutive patients who underwent ossiculoplasty were included. Those without 3 months postoperative audiogram were excluded. Cases, where the endoscope was the only instrument used, were classed as TEES, and others were as microsurgery.

\subsection{Variables}


Apart from the surgical approach, other variables analysed included the condition of the ossicles, type and the material used for ossiculoplasty and tympanic membrane graft.

\subsection{Ethical considerations}

The study is registered as an audit and further ethics committee approval was not required.

\subsection{Surgical details}

Operations were carried out using either a Zeiss microscope or a 0-degree, $3 \mathrm{~mm}, 14 \mathrm{~cm}$ Storz endoscope in the TEES or combined group. The disease was characterized at tympanotomy including perforations, condition of ossicles, and the site and extent of cholesteatoma before completion of ossiculoplasty.

\subsection{Audiology outcomes}

Audiometric outcomes including air conduction (AC), bone conduction (BC), and air-bone gap (ABG) were recorded at 3- and 12- months postoperatively and compared to pre-operative levels. The average of 500, 1000, 2000, and $3000 \mathrm{~Hz}$ was used for analysis. as per AAOHNS recommendations.

\subsection{Outcome measures}

The primary outcome was postoperative ABG. Secondary outcomes included changes in AC, BC, and ABG of less than $20 \mathrm{~dB}$.

\subsection{Statistical analysis}

The data was compiled using the Common Otology Audit database and analysed using SPSS 27. Data are summarised as means +/- standard deviation (SD) for numerical values and count and percentages for categorical variables. The student t-test was used for continuous data and categorical variables were compared using the chi-square and Fischer's exact test as appropriate. A p-value of $<0.05$ was considered statistically significant.

\section{Results}

\subsection{Patient Population and Demographic Data}

The mean age was 36 years (range: 7-82) and 71 (56\%) patients were male. Fifty patients (31.8\%) underwent TEES whereas 107 (68.2\%) underwent microsurgery (80) or a combined endoscopic approach (27).

The mean (SD in brackets) pre-operative hearing for the whole group, microscopic group, and TEES group were as follows. AC $43.4 \mathrm{~dB}(17.7), 42.7 \mathrm{~dB}$ (18.1), 44.8dB (16.8). BC 20.3dB (12.3), 19.8dB (12.7), and $21.2 \mathrm{~dB}$ (11.4) and the ABG 21.8dB (11.8), 21dB (12.3) 23.6dB (10.4), respectively. There was no significant difference between groups ( $\mathrm{p}>0.05)$.

\subsection{Disease characteristics}

Nineteen $(12.1 \%)$ of the whole cohort were noted to have stapes erosion, 11 of these patients were dealt with TEES (22\% of the TEES) compared to only $8(7.5 \%)$ in the microscopic group $(\mathrm{P}<0.001)$. Other disease characteristics such as tympanic membrane perforation, condition of ossicles, site, and extent of cholesteatoma are shown in Table 1.

\subsection{Ossiculoplasty type}

Cartilage ossiculoplasty was the commonest approach, with a cartilage disc applied directly to stapes head touching the tympanic membrane in $71(45.5 \%)$ patients, $47(44.8 \%)$ within the microscopic group and 24 (48\%) of the TEES group, their proportion did not differ significantly $(\mathrm{P}>0.05)$. The other materials used were incus in 42 ears (27.1\%), Titanium PORP / TORP (Grace medical, USA) in 34 (21.9\%), malleus head, bone cement and bone chip in very small cases (Table 2) Tympanic membrane to stapes ossiculoplasty was the commonest type of procedure employed, in 128 (82.1\%) of the whole cohort, 93 of those were approached microscopically and 35 via TEES. The other ossiculoplasty types were tympanic membrane to footplate with 
TORP and footplate shoe in $11(7.1 \%)$ and incus to stapes in $14(9 \%)$. There was no significant difference in the proportion with each type of reconstruction (Table 2)

\subsection{Tympanoplasty materials}

Significantly more patients within the microsurgery group had temporalis fascia graft compared to the TEES group, $92(86 \%)$ Vs $1(2 \%), \mathrm{p}<0.001$, perichondrium and cartilage was the preferred graft within the TEES group, $33(66 \%)$ v $2(1.9 \%), \mathrm{p}<0.001$. Details of intra-operative characteristics can be found in Table 2 .

\subsection{Hearing outcomes}

There were significant improvements at 3-month follow-up in AC, 43.4dB (17.7) Vs $36.2 \mathrm{~dB}$ (17.4), p $<0.001$, BC, 20.3dB (12.3) Vs 17.6dB (12.6), p<0.001, and ABG 21.8dB, (11.8) Vs 16.7dB (10.4), p<0.001, compared to pre-operative hearing.

In the microsurgery group, there were statistically significant improvements in AC, $42.7 \mathrm{~dB}$ (17.7) Vs 36dB (17.2), $\mathrm{p}<0.001)$ ), BC, 19.8dB (12.3) Vs 16.7dB (12.4), p<0.001 and ABG (21dB (11.8) Vs 16.9dB (10.4), $\mathrm{p}<0.001$. In the TEES group there were statistically significant improvements in $\mathrm{AC}, 44.8 \mathrm{~dB}(16.8) \mathrm{Vs}$ $36.8 \mathrm{~dB}(17.8), \mathrm{p}<0.001, \mathrm{BC} 21.2 \mathrm{~dB}(11.4)$ Vs $19.5 \mathrm{~dB}$ (12.9), $\mathrm{p}<0.001$, and ABG 23.6dB (10.4) Vs $16 \mathrm{~dB}$ (10.40), $\mathrm{p}<0.001$.

When compared to the microscopic approach, there was no statistically significant difference in improvement in $\mathrm{AC}, 6.7 \mathrm{~dB}(19.2) \mathrm{Vs} 8.0 \mathrm{~dB}$ (14.9), $\mathrm{p}=0.665, \mathrm{BC}, 3.1 \mathrm{~dB}$ (10.4) Vs $1.7 \mathrm{~dB}$ (6.6), $\mathrm{p}=0.632$ and $\mathrm{ABG} 4.0 \mathrm{~dB}$ (14.8) Vs $7.5 \mathrm{~dB}$ (13.6), $\mathrm{p}=0.160$ in those who underwent TEES.

$73 \%$ of those in the microscopic group and $72 \%$ in the TEES group achieved an ABG better than $20 \mathrm{~dB}$ with no significant difference $(\mathrm{p}=0.907)$. All variables including the use of different TM grafts and ossiculoplasty materials made no significant difference to the AC, BC and ABG (all comparisons $\mathrm{p}>0.05$ ) across the whole cohort and when hearing outcomes of the microscopic and TEES groups were compared (see Table 3)

Nineteen of 157 patients were noted to have stapes dehiscence at the time of operation. Both microscopic and TEES sub-groups achieved improvements in AC, 6.2dB (16.8) Vs 7.2dB (18.1), p=0.8, BC 1.6dB (5.4) Vs $2.8 \mathrm{~dB}(9.8), \mathrm{p}=0.62$ and $\mathrm{ABG}, 4.6 \mathrm{~dB}(15) \mathrm{Vs} 5.2 \mathrm{~dB}(14.4)$ and the difference was not statistically significant $(\mathrm{p}=0.85)$. This remained true when comparing those approached microscopically and via TEES $(\mathrm{p}>0.05)$ (see Table 4)

Of 79 patients (59 microscopic and 20 TEES) who were reviewed 12 months post-ossiculoplasty, there were no significant changes in AC, BC, or ABG when compared to 3 months ( $\mathrm{p}>0.05$ ). (Table 3)

\subsection{Secondary outcomes}

At 3-month follow-up, 3.7\% and $4 \%$ had residual perforations $(\mathrm{p}=0.623)$ and ear discharge was observed in $5.6 \%$ and $2 \%(\mathrm{P}=0.286)$ of the microscopic and TEES groups, respectively. There was a higher incidence of myringitis in $38 \%$ of the TEES group when compared to $22.4 \%$ in the microsurgery group $(\mathrm{p}=0.042)$. Only one patient was noted to have residual cholesteatoma and they were part of the microscopic group $(\mathrm{p}=0.682)$. There were no statistically significant differences in postoperative complications between both groups at 12 months. (Table 3 ).

\section{Discussion}

To our knowledge, our study is the biggest series comparing ossiculoplasty by microscopic route to that of TEES performed by a single surgeon and has demonstrated that it produces comparable hearing outcomes in $\mathrm{AC}, \mathrm{BC}$ and $\mathrm{ABG}$ less than $20 \mathrm{~dB}$

There was no significant difference in the improvement in AC, BC, and ABG of the 93 patients undergoing TM-stapes reconstruction via the microscopic approach compared to 35 patients in the TEES group. Our study also showed that there was also no significant difference with various tympanic membrane grafts and ossiculoplasty materials including just using cartilage as opposed to the use of titanium PORP and incus 
transposition. These results were comparable to those obtained by Potsangbam et ${ }^{4}$ al in their cases with cartilage ossiculoplasty with intact stapes superstructure.

Only 19 patients were noted to have stapes dehiscence at the time of operation. Despite this, all patients achieved improvement in AC, BC, and ABG. There was no statistical difference in hearing improvement between those 19 patients with dehiscence compared to those 138 without, approached either microscopically or via TEES.

There are some studies in the literature ${ }^{\mathbf{5 - 9}}$, including an RCT of 118 cases comparing TEES and microsurgery using PORP and TORP showing similar results between microsurgery and TEES. But unlike our series, they were not by a single surgeon and haven't included cartilage and incus grafts.

Our study has certain limitations. The patients who were operated on before 2014 all had a microscopic approach before the surgeon started TEES. In addition, there was no randomization of patients to groups, therefore the potential for selection bias exists. But the advantages to our study are prospective data collection and all surgeries being conducted by a single surgeon, thus eliminating some of the bias.

\section{Conclusion}

We demonstrated improvement in audiometric outcomes regardless of surgical approach, stapes condition, type of ossiculoplasty, or TM reconstruction material used. TEES is therefore safe and as effective as microsurgery in ossiculoplasty and has the advantages of less pain ${ }^{\mathbf{1 0}}$ and morbidity. Larger sample size and a longer follow-up period may show significant discrimination between surgical approaches and materials used.

\section{Key Points}

1. Results of ossiculoplasty via trans-canal endoscopic (TEES) route is comparable to that of the traditional microscopic approach

2. There was no difference in complications or secondary outcomes such as tympanic membrane residual perforation or ear discharge between these groups

3. Both groups achieved an air-bone gap of less than $20 \mathrm{~dB}$ in more than $70 \%$ of the cases

4. In our experience there was no difference in outcomes based on ossiculoplasty material such as cartilage, titanium prosthesis or autologous grafts such as incus.

5. Eroded stapes superstructure does not preclude getting improvements in ABG of less than 20dB, but larger studies with longer follow-ups might be useful.

\section{References}

1. Kobayashi T, Gyo K, Komori M, Hyodo M. Efficacy and Safety of Transcanal Endoscopic Ear Surgery for Congenital Cholesteatomas: A Preliminary Report. Otol Neurotol. 2015 Dec;36(10):1644-50. doi: 10.1097/MAO.0000000000000857. PMID: 26485586.

2. Li B, Zhou L, Wang M, Wang Y, Zou J. Endoscopic versus microscopic surgery for treatment of middle ear cholesteatoma: A systematic review and meta-analysis. Am J Otolaryngol. 2021 MarApr;42(2):102451. doi: 10.1016/j.amjoto.2020.102451. Epub 2020 Mar 5. PMID: 33360773.

3. Hoskison EE, Harrop E, Jufas N, Kong JHK, Patel NP, Saxby AJ. Endoscopic Stapedotomy: A Systematic Review. Otol Neurotol. 2021 Dec 1;42(10):e1638-e1643. doi: 10.1097/MAO.0000000000003242. PMID: 34267093.

4. Potsangbam DS, Akoijam BA. Endoscopic Transcanal Autologous Cartilage Ossiculoplasty. Indian J Otolaryngol Head Neck Surg. 2019 Mar;71(1):54-59. doi: 10.1007/s12070-018-1518-x. Epub 2018 Oct 30. PMID: 30906714; PMCID: PMC6401045.

5. Caloway CL, Basonbul RA, Ronner EA, Tolisano AM, Zhu AW, Suresh H, Lee DJ, Isaacson B, Cohen MS. Pediatric Endoscopic Ossiculoplasty Following Surgery for Chronic Ear Disease. Laryngoscope. 2020 Dec;130(12):2896-2899. doi: 10.1002/lary.28526. Epub 2020 Feb 5. PMID: 32022283. 
6. Das A, Mitra S, Ghosh D, Sengupta A. Endoscopic ossiculoplasty: Is there any edge over the microscopic technique? Laryngoscope. 2020 Mar;130(3):797-802. doi: 10.1002/lary.28074. Epub 2019 May 23. PMID: 32073145.

7. Yawn RJ, Hunter JB, O'Connell BP, Wanna GB, Killeen DE, Wick CC, Isaacson B, Rivas A. Audiometric Outcomes Following Endoscopic Ossicular Chain Reconstruction. Otol Neurotol. 2017 Oct;38(9):1296-1300. doi: 10.1097/MAO.0000000000001527. PMID: 28796085.

8. Tsetsos N, Vlachtsis K, Stavrakas M, Fyrmpas G. Endoscopic versus microscopic ossiculoplasty in chronic otitis media: a systematic review of the literature. Eur Arch Otorhinolaryngol. 2021 Apr;278(4):917-923. doi: 10.1007/s00405-020-06182-6. Epub 2020 Jul 7. PMID: 32632614.

9. Chung J, Kang JY, Kim MS, Kim B, Choi JW. Microscopic vs Endoscopic Ear Surgery for Congenital Ossicular Anomaly. Otolaryngol Head Neck Surg. 2020 Apr;162(4):548-553. doi: 10.1177/0194599819900489. Epub 2020 Jan 21. PMID: 31959042.

10. McCallum R, Mohd Slim MA, Iyer A. An audit of post-operative pain scores after endoscopic and microscopic ear surgery. Clin Otolaryngol. 2021 Dec 20. doi: 10.1111/coa.13904. Epub ahead of print. PMID: 34931457.

11.

Table 1. This table shows the operative findings and pre-operative audiological results in both TEES and microsurgery groups. There was no difference in pre-operative hearing levels between groups, cholesteatoma was more extensive in the microscopic group. Table 2. This shows the types of ossiculoplasty reconstruction, materials used in ossiculoplasty and the tympanic membrane graft. Cartilage was the commonest material used followed by incus and titanium PORP / TORP and there was no difference between groups. There was a significant difference in the tympanic membrane graft material, with more cases in the microscopic group using temporalis facia $(\mathrm{p}<0.001)$ Table 3 . This shows the outcomes at 3 and12 months following surgery. There was no significant difference in hearing or other outcomes apart from a higher incidence of myringitis in the TEES group at 3 months $(\mathrm{p}=0.042)$. At 12 months following surgery, there was a significantly lower $\mathrm{AC}$ level in the TEES group $(\mathrm{p}=0.033)$. All the other outcomes did not show any significant difference.Table 4. This shows the mean differences of AC, BC and ABG between those with and without erosion of stapes superstructure. There was no statistically significant difference between groups.

\section{Hosted file}

Ossic Table 1.docx available at https://authorea.com/users/456980/articles/553867-comparisonof-endoscopic-vs-microscopic-ossiculoplasty-a-study-of-157-consecutive-cases

\section{Hosted file}

Ossic Table 2.docx available at https://authorea.com/users/456980/articles/553867-comparisonof-endoscopic-vs-microscopic-ossiculoplasty-a-study-of-157-consecutive-cases

\section{Hosted file}

Ossic Table 3.docx available at https://authorea.com/users/456980/articles/553867-comparisonof-endoscopic-vs-microscopic-ossiculoplasty-a-study-of-157-consecutive-cases

\section{Hosted file}

Ossic Table 4.docx available at https://authorea.com/users/456980/articles/553867-comparisonof-endoscopic-vs-microscopic-ossiculoplasty-a-study-of-157-consecutive-cases 